\title{
Effect of azelastine on sulphur dioxide induced impairment of ciliary motility in airway epithelium
}

J Tamaoki, A Chiyotani, N Sakai, K Takeyama, K Konno

\begin{abstract}
Objective-The effect of azelastine on airway mucociliary transport function was studied by measuring ciliary motility of human bronchial epithelium in vitro with a photoelectric method.

Method-Bronchial epithelial cells were obtained by fibreoptic bronchoscopy, mounted in a Rose chamber, and perfused with Krebs-Henseleit solution. The preparations were placed on a microscope stage equipped with an illuminator, and the variations of light intensity caused by ciliary beating were detected by a photometer.

Results-The addition of azelastine to the perfusate increased ciliary beat frequency (CBF) in a dose dependent manner without ciliary discoordination. The mean (SE) maximal increase from the baseline value and the concentration required to produce a half maximal effect were $27 \cdot 0(4 \cdot 2) \%$ and $9.2 \times 10^{-6}$ mol/h, respectively. Exposure of the cells to the perfusate containing $3 \mathrm{ppm}$ sulphur dioxide rapidly decreased CBF by $59 \cdot 2(5 \cdot 0) \%$, and was accompanied by a reduction in intracellular cyclic AMP levels from $38 \cdot 1(4 \cdot 3)$ to $10 \cdot 1(2 \cdot 4)$ pmol/mg protein. This effect was prevented by pretreatment of cells with azelastine in a dose dependent manner.

Conclusions-Azelastine not only stimulates ciliary motility of airway epithelium and hence mucociliary transport function, but may also protect against sulphur dioxide induced ciliary dysfunction, probably by inhibiting intracellular cyclic AMP loss.
\end{abstract}

First Department of Medicine, Tokyo

Women's Medical

College, 8-1 Kawada-

cho, Shinjuku, Tokyo

162, Japan

J Tamaoki

A Chiyotani

N Sakai

K Takeyama

K Konno

Reprint requests to:

Dr J Tamaoki

Received 30 July 1992

Returned to authors

22 October 1992

Revised version received

20 November 1992

Accepted 4 December 1992

\section{(Thorax 1993;48:542-546)}

Removal of cellular debris, inhaled particles, and bacteria from the conducting airways of the respiratory tract are important factors in the defence mechanism of the lung. Movement of mucus containing adherent materials is directed posteriorly from the nasal cavity and upward from the lung, converging at the pharynx. This transport function is dependent on the beat frequency and coordination of cilia, mucus production, and the rheological properties of mucus.

Several bronchial disorders including chronic bronchitis, asthma, and cystic fibrosis are associated with an impairment of mucociliary clearance, ${ }^{2-4}$ and exposure to various noxious agents such as cigarette smoke, ammonia, formaldehyde, and sulphur dioxide $\left(\mathrm{SO}_{2}\right)$ can result in inhibition of ciliary function or ciliostasis. ${ }^{56}$

Azelastine, 4-(p-chlorobenzyl)-2-(hexahydro-1-methyl-1 H-azepin-4-yl)-1-(2H)phthalazione hydrochloride, is an orally effective and long lasting antiallergic drug that prevents bronchospasm by inhibiting histamine release from mast cells ${ }^{7}$ and leukotriene synthesis in the lung. ${ }^{8}$ In addition, this drug possesses anti-inflammatory actions including suppression of superoxide generation by leukocytes, ${ }^{9}$ prostaglandin release from macrophages, ${ }^{10}$ and microvascular leakage. ${ }^{11}$ The purpose of the present study was (a) to characterise the $\mathrm{SO}_{2}$ induced inhibition of airway ciliary motility; (b) to determine whether azelastine can protect against airway mucociliary dysfunction; and (c) if so, to investigate its mechanism of action. We therefore studied ciliary motility of human airway epithelium in vitro by a photoelectric method.

\section{Methods}

TISSUE PREPARATION

Fibreoptic bronchoscopy including transbronchial lung biopsy was carried out on 24 patients with a peripheral lung cancer. None had a history of respiratory tract infection for at least one month and any medication was stopped 14 days before the bronchoscopy. After topical anaesthesia of the upper airway with 4\% lignocaine, a fibreoptic bronchoscope (Type 1T20; Olympus, Tokyo, Japan) was inserted transorally through an endotracheal tube. A standard bronchial brush (BC9C; Olympus) was passed through the biopsy channel and epithelial cells were scraped at three different levels: the carina of the right upper lobe; the orifice of the middle lobe; and in a segment of the right lower lobe. Collection of samples was restricted to areas free of obvious mucosal invasion by carcinoma or acute inflammation, swelling, pus, or reddening. The cells were extracted from the brush by brisk agitation in a petri dish containing Krebs-Henseleit solution of the following composition (in $\mathrm{mmol} / \mathrm{l}$ ): $\mathrm{NaCl}, 118$; $\mathrm{KCl}, 5 \cdot 9 ; \mathrm{CaCl}_{2}, 2 \cdot 5 ; \mathrm{MgSO}_{4}, 1 \cdot 2 ; \mathrm{NaH}_{2} \mathrm{PO}_{4}$ 1.2; $\mathrm{NaHCO}_{3}, 25 \cdot 5$; glucose, 5.6. The cell 
suspension was then transferred to a Rose chamber ${ }^{12}$ for the measurement of ciliary beat frequency $(\mathrm{CBF})$ within 30 minutes.

\section{MEASUREMENT OF CBF}

To measure CBF of the airway epithelium a microscope (Optiphoto-XF; Nikon, Tokyo) equipped with a trinocular body, a phase contrast condenser, and an on base type of halogen illuminator were used..$^{13} 14$

The photometer (NFX-II; Hamamatsu Photonics, Hamamatsu, Japan) with an inbuilt periplanatic eyepiece, a limiting aperture, and a lateral focusing telescope was attached to the head of the microscope. The Rose chamber containing epithelial cells was set on a microscope stage maintained at $37^{\circ} \mathrm{C}$ by an air stream stage incubator (E5B2; Omron, Tokyo) and perfused with warmed Krebs-Henseleit solution aerated with $95 \%$ $\mathrm{O}_{2}$ and $5 \% \mathrm{CO}_{2}$ at a constant pressure of 20 $\mathrm{cm} \mathrm{H}_{2} \mathrm{O}$.

Light from the illuminator passed through the preparation in varying intensities because of the beating action of the cilia; this was viewed directly at a magnification of $\times 400$. Variations of light intensity were detected by the photometer and transduced to voltage impulses. These electrical signals were amplified, displayed on the oscilloscope screen (LBO-522; Leader, Tokyo), and recorded by a pen recorder (VP6213; Panasonic, Tokyo). Throughout the experiment, the same group of cilia in each preparation was studied - that is, the relative position of the diaphragm of the photometer and the selected epithelial border were kept constant. The choice of the ciliated area was randomised by using an eyepiece graticule and selecting the group of cilia which lay closest to the crosspiece of the graticule on the equatorial line. The longitudinal axis of the field of the photometer was viewed perpendicular to the cell border and the measurements of $\mathrm{CBF}$ were averaged from clumps of two or more cells with borders free of debris. The mean intracell coefficient of variation of $\mathrm{CBF}$, defined as the ratio of the standard deviation to the mean value as a percentage, was less than $5 \%$. There were no significant differences in $\mathrm{CBF}$ among epithelial cells obtained from three different levels of the airway.

In addition to CBF measurements, ciliary coordination was assessed by the image of beating recorded on a video camera (Type 1864-01; Hamamatsu Photonics) with a 0.75 inch video cassette recorder (VO-5800; Sony, Tokyo) capable of freeze frame replay. Ciliary discoordination was defined as the loss of the metachronal wave on the free border of the cell clump. ${ }^{15}$

\section{STUDY DESIGN}

The preparation was allowed to stabilise for 30 minutes before the measurement of CBF. After determination of baseline CBF, azelastine (Eizai Company, Tokyo) in a concentration of $10^{-4} \mathrm{~mol} / 1$ was added to the perfusate and CBF was continuously recorded for the next 20 minutes. For time controls, measure- ments were obtained when only KrebsHenseleit solution was used for perfusion. To generate the $\mathrm{CBF}$ dose-response curves, azelastine was cumulatively added in half molar increments from $10^{-7}$ to $10^{-3} \mathrm{~mol} / \mathrm{l}$, and the response to a given concentration was allowed to reach a plateau before proceeding to the next higher concentration.

To test any protective action of azelastine on ciliary dysfunction, we examined the effect of pretreatment of the cells with azelastine on the $\mathrm{SO}_{2}$ induced decrease in CBF. The preparation was incubated with $10^{-6} \mathrm{~mol} / \mathrm{l}$ azelastine for 20 minutes. When the CBF response reached a plateau, Krebs-Henseleit solution with a mixture of $95 \% \mathrm{O}_{2}, 5 \% \mathrm{CO}_{2}$ and $3 \mathrm{ppm} \mathrm{SO}$ bubbling through it perfused the cells and azelastine mixture for $20 \mathrm{~min}$ utes. In this series of experiments, cells were preincubated with various concentrations of azelastine at $10^{-7}$ to $10^{-5} \mathrm{~mol} / 1$ and the responses of $\mathrm{CBF}$ to $\mathrm{SO}_{2}$ were determined in order to assess a dose-response relationship.

\section{MEASUREMENT OF CYCLIC AMP}

After 20 minutes exposure of epithelial cells to azelastine alone, $\mathrm{SO}_{2}$ alone, or $\mathrm{SO}_{2}$ plus azelastine, preparations were quickly removed from the chambers and sonicated with a bath type sonicator (PT-101; Sino Company, Tokyo) in ice cold $10 \%$ trichloroacetic acid. After the extraction of trichloroacetic acid with ether, the residue was dissolved in acetate buffer. The cyclic AMP levels were measured in duplicate by $\left[{ }^{3} \mathrm{H}\right]$ cyclic AMP (New England Nuclear, Boston, Massachusetts, USA), ${ }^{16}$ corrected for ether extraction of $86 \%$ recovery, and normalised for protein content of the cells by the Lowry method $^{17}$ with bovine serum albumin as a standard.

\section{STATISTICAL ANALYSES}

The effects of azelastine and $\mathrm{SO}_{2}$ on $\mathrm{CBF}$ were compared with the appropriate time controls (Krebs-Henseleit solution alone) using Friedman's two way analysis of variance. Comparisons between the effects of $\mathrm{SO}_{2}$ with or without azelastine were made with the Kruskal-Wallis one way analysis of variance test. A different donor of ciliated cells was used in each experiment; $n$ refers to the number of patients from which samples were obtained, and a $p$ value of $<0.05$ was considered significant.

\section{Results}

There was no significant difference in baseline CBF between the cells treated with azelastine and the Krebs-Henseleit solution time controls. The CBF did not change significantly during a 20 minute observation period in the control experiment, whereas addition of azelastine $\left(10^{-4} \mathrm{~mol} / \mathrm{l}\right)$ rapidly increased the mean (SE) CBF from $17 \cdot 2(0 \cdot 7) \mathrm{Hz}$ to a peak value of $21.8(1.2) \mathrm{Hz}$ within five minutes, being significantly faster than the control $(\mathrm{p}<0.05, \mathrm{n}=11)$, and remained faster thereafter (fig 1). The stimulatory effect of 
Figure 1 Effect of azelastine on ciliary beat frequency $(C B F)$ of human bronchial epithelium. Azelastine $\left(10^{-4} \mathrm{~mol} / \mathrm{l}\right)$ was added to the perfusate at time 0 (arrow). Bar lines indicate $1 S E(n=11$ for each group). ${ }^{*} p<0.05 \mathrm{v}$

Krebs-Henseleit controls.

Figure 2 Concentration dependent effect of azelastine on ciliary beat frequency $(C B F)$ of human bronchial epithelium. Azelastine was cumulatively added to the perfusate in half molar increments. Data are expressed as percentage of the baseline CBF obtained before addition of azelastine. Bar lines indicate $1 S E$ ( $n=9$ for each group). ${ }^{*} p<0.05 \mathrm{v}$ Krebs-Henseleit controls.

Figure 3 Effect of azelastine on intracellular cyclic AMP levels of human bronchial epithelium. The cells were incubated with various concentrations of azelastine for 20 minutes and the cyclic AMP concentrations were determined by radioimmunoassay. Bar lines indicate $1 S E(n=$ for each bar). ${ }^{*} p<0.05$, for each bar)

Krebs-Henseleit controls.
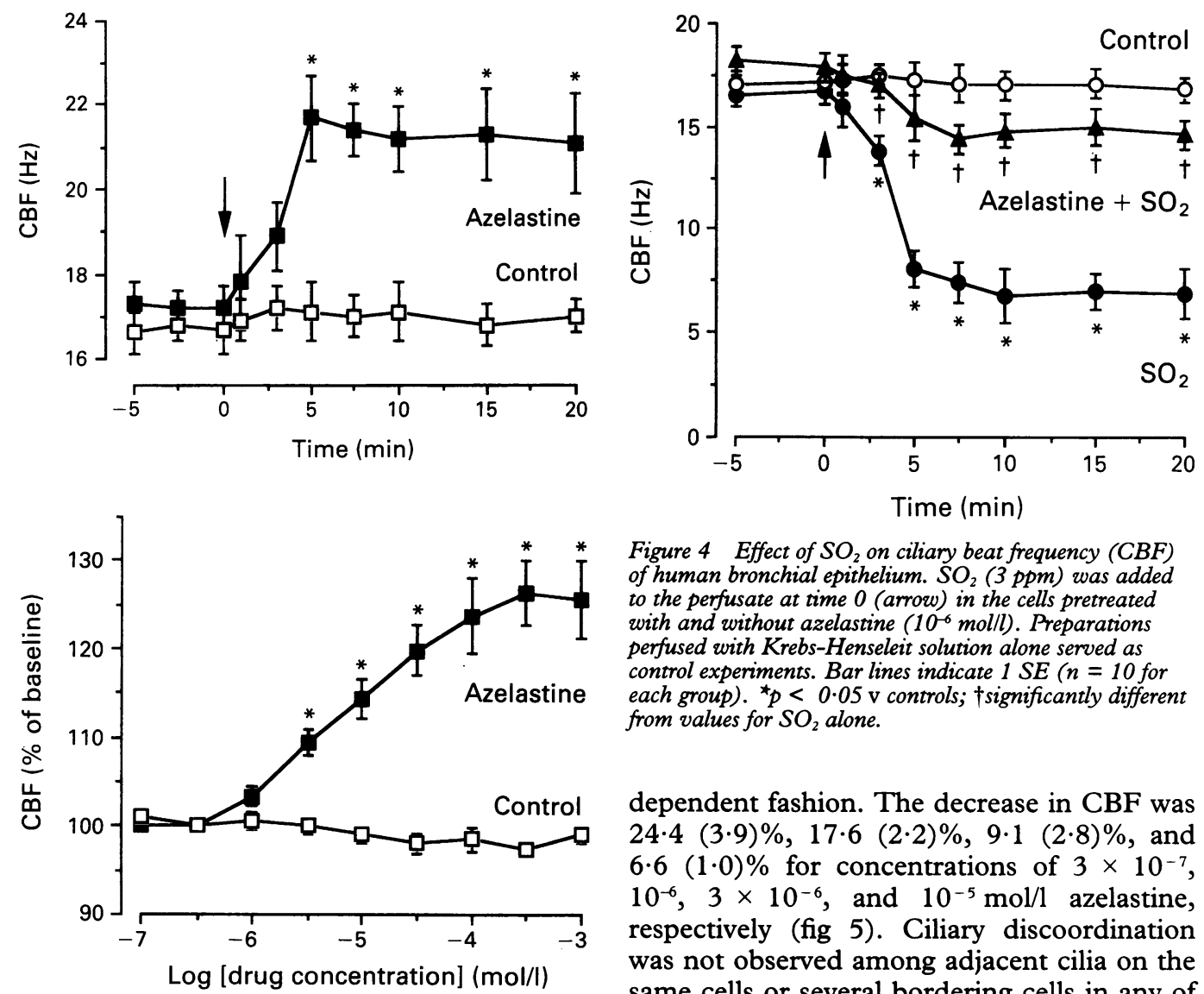

azelastine on the cilia was dose dependent with the maximal increase from the baseline $\mathrm{CBF}$ and the concentration required to produce a half maximal effect being $27 \cdot 0(4 \cdot 2) \%$ $(\mathrm{p}<0.05, \mathrm{n}=9)$ and $9.2 \times 10^{-6} \mathrm{~mol} / \mathrm{l}$, respectively (fig 2 ). This was accompanied by corresponding increases in intracellular cyclic AMP levels (fig 3) where the threshold concentration of azelastine $\left(3 \times 10^{-6} \mathrm{~mol} / \mathrm{l}\right)$ was identical to that for ciliary stimulation.

The addition of $3 \mathrm{ppm} \mathrm{SO}_{2}$ to the perfusate caused a rapid reduction of ciliary motility, with the CBF values significantly slower than the control experiments at $2 \cdot 5-20$ minutes after the initiation of $\mathrm{SO}_{2}$ exposure $(\mathrm{p}<0.05, \mathrm{n}=10)$, an effect that was significantly inhibited by pretreatment of cells with $10^{-6} \mathrm{~mol} / \mathrm{l}$ azelastine (fig 4). The maximal decrease in CBF from the baseline value induced by $\mathrm{SO}_{2}$ was $59.2(5.0) \%$, and azelastine protected against this inhibition in a dose

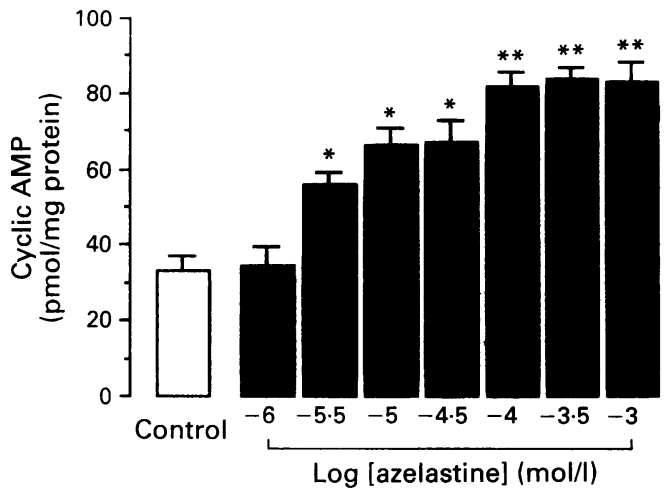

Figure 4 Effect of $\mathrm{SO}_{2}$ on ciliary beat frequency (CBF) of human bronchial epithelium. $\mathrm{SO}_{2}$ (3 ppm) was added to the perfusate at time 0 (arrow) in the cells pretreated with and without azelastine $\left(10^{-6} \mathrm{~mol} / \mathrm{l}\right)$. Preparations perfused with Krebs-Henseleit solution alone served as control experiments. Bar lines indicate $1 S E(n=10$ for each group). ${ }^{*} p<0.05 \mathrm{v}$ controls; $\dagger$ significantly different from values for $\mathrm{SO}_{2}$ alone.

dependent fashion. The decrease in $\mathrm{CBF}$ was $24.4(3.9) \%, 17.6(2.2) \%, 9 \cdot 1(2.8) \%$, and $6.6(1.0) \%$ for concentrations of $3 \times 10^{-7}$, $10^{-6}, 3 \times 10^{-6}$, and $10^{-5} \mathrm{~mol} / 1$ azelastine, respectively (fig 5). Ciliary discoordination was not observed among adjacent cilia on the same cells or several bordering cells in any of the experiments.

Exposure of epithelial cells to $\mathrm{SO}_{2}$ decreased intracellular cyclic AMP levels from $38 \cdot 2(4 \cdot 3)$ to $10 \cdot 1(2 \cdot 4) \mathrm{pmol} / \mathrm{mg}$ protein $(p<0.01, n=12)$. Azelastine pretreatment prevented this effect in a dose dependent manner so that the reduction of cyclic AMP levels was completely reversed at concentrations of $3 \times 10^{-6}$ and $10^{-5} \mathrm{~mol} / 1$ (fig 6).

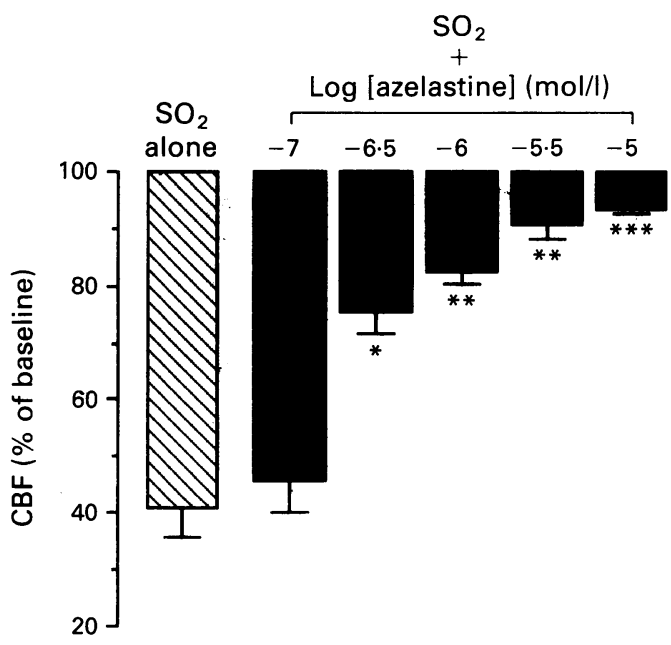

Figure 5 Concentration dependent effect of azelastine on the $\mathrm{SO}_{2}$ induced decrease in ciliary beat frequency $(C B F)$ of human bronchial epithelium. The cells were pretreated with various concentrations of azelastine for

20 minutes and exposed to Krebs-Henseleit solution containing $\mathrm{SO}_{2}$. Data are expressed as percentage of the baseline $\mathrm{CBF}$ obtained before $\mathrm{SO}_{2}$ exposure. $B$ ar lines indicate $I S E\left(n=9\right.$ for each bar). ${ }^{\star} p<0.05$,

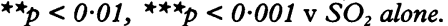


Figure 6 Concentration dependent effect of azelastine on the $\mathrm{SO}_{2}$ induced decrease in intracellular cyclic AMP levels of human bronchial epithelium. The cells were pretreated with various concentrations of azelastine for 20 minutes and exposed to Krebs-Henseleit solution containing $\mathrm{SO}_{2 .}$ Bar lines indicate $1 S E(n=12$ for each bar). ${ }^{\star *} p<0.01 \mathrm{v}$ Krebs-Henseleit controls; $\dagger p<0.05,+\dagger \dagger p<0.001 \mathrm{v}$ $\mathrm{SO}_{2}$ alone.

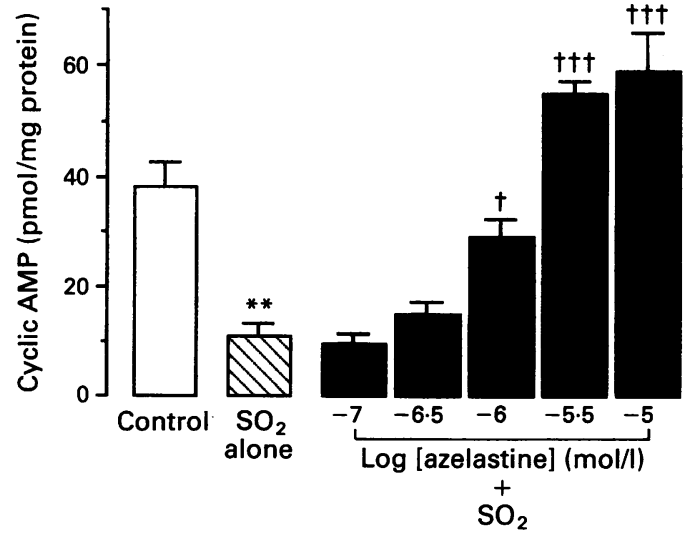

\section{Discussion}

We have shown that azelastine stimulates ciliary motility of human bronchial epithelium and protects against inhibition of cilia by $\mathrm{SO}_{2}$, presumably by the accumulation of intracellular cyclic AMP.

It has been shown that ciliary motility in epithelial cells is regulated by various endogenous determinants including cyclic $\mathrm{AMP},{ }^{13}$ $\mathrm{Ca}^{2+},{ }^{18}$ and protein kinase $\mathrm{C} .{ }^{19}$ In the present study azelastine increased CBF of airway epithelium in a dose dependent fashion, the concentration required to produce a half maximal effect $\left(9.2 \times 10^{-6} \mathrm{~mol} / \mathrm{l}\right)$ being similar to that previously found to inhibit histamine release in mast cells $\left(5 \times 10^{-6}\right.$ $\mathrm{mol} / \mathrm{l})^{20}$ and to inhibit platelet activating factor release in macrophages $\left(1.6 \times 10^{-5}\right.$ $\mathrm{mol} / \mathrm{l}){ }^{21}$ Azelastine also raised intracellular cyclic AMP levels, as shown by Akagi et al, ${ }^{22}$ which suggests that azelastine causes cyclic AMP to accumulate in mast cells. Increased availability of cyclic AMP may activate glycogenolysis and enhance the production of ATP (an immediate energy source for the mechanical activity of the living cilia $^{23}$ ) through the Krebs cycle, thereby stimulating ciliary activity.

Mucociliary interaction and hence mucus transport in the airway is governed by ciliary movement, airway patency, mucus production, and airway fluid dynamics. ${ }^{1}$ Among these factors, ciliary motility of airway epithelial cells has been suggested to play a key part in mucociliary clearance. Moreover, the effectiveness of ciliary action depends on several characteristics of ciliary beating of which the $\mathrm{CBF}$ is but one. The coordination of the beating pattern, for example, is also important in ciliary performance. In the present study neither ciliary discoordination nor mucus production was observed throughout the experiments. We believe therefore that the increase in CBF induced by azelastine may result in enhanced mucociliary transport as predicted by theoretical models of mucociliary pumping. ${ }^{24}$

Mucociliary clearance in the respiratory tract has been known to be impaired in various airway diseases such as chronic bronchitis, asthma, and cystic fibrosis, ${ }^{2-4}$ and by the inhalation of several noxious agents. ${ }^{56}$ Dalhamn ${ }^{5}$ reported that $\mathrm{SO}_{2}$ inhibited ciliary activity of rat tracheal epithelial cells but its mechanism of action was unknown. We found in human bronchial epithelium that exposure to $\mathrm{SO}_{2}$ likewise decreased $\mathrm{CBF}$ and that this effect was accompanied by a decrease in intracellular cyclic AMP content. The $\mathrm{SO}_{2}$ induced interference with ciliary activity may therefore be at least in part due to the impairment of cyclic AMP production. It remains to be determined, however, whether $\mathrm{SO}_{2}$ acts directly on the cyclic AMP dependent pathway in the epithelium or indirectly by stimulating cilioinhibitory mediators such as adenosine ${ }^{14}$ and oxygen radicals. ${ }^{25}$

Recent evidence suggests that the agent capable of elevating cyclic AMP in airway epithelium can prevent lung injury. ${ }^{26}$ In this study pretreatment of epithelial cells with azelastine at concentrations insufficient to increase intracellular cyclic AMP levels by itself $\left(3 \times 10^{-7} \mathrm{~mol} / \mathrm{l}, 10^{-6} \mathrm{~mol} / \mathrm{l}\right)$ reversed the $\mathrm{SO}_{2}$ induced reduction of $\mathrm{CBF}$ and cyclic AMP levels. We therefore speculate that this reversal may be produced by the inhibition of cyclic AMP loss.

In conclusion, the present studies indicate that the antiallergic drug azelastine not only enhances the beating action of human bronchial cilia but also protects against the impairment of ciliary motility, and suggest that this drug could be of value in patients with mucociliary dysfunction.

We thank Yoshimi Sugimura and Masayuki Shino for their technical assistance.

This study was supported in part by a grant for scientific research No. 63770524 from the Ministry of Education, Science and Culture of Japan.

1 Wanner A. Clinical aspects of mucociliary transport. $A m$ Rev Respir Dis 1977;116:73-125.

2 Hilding AC. Mucociliary insufficiency and its possible relation to chronic bronchitis and emphysema. Med Thorac 1965;22:329-45.

3 Iravani J, Melville GN. Mucociliary function in the respiratory tract as influenced by physicochemical factors. Pharmacol Ther 1976;2:471-92.

4 Mezey RJ, Cohn MA, Fernandez J, Januszkiewicz AJ, Wanner A. Mucociliary transport in allergic patients with antigen-induced bronchospasm. Am Rev Respir Dis 1978;118:677-84.

5 Dalhamn T. Studies on the effect of sulphur dioxide on ciliary activity in rabbit trachea in vivo and in vitro and on the resorptional capacity of the nasal cavity. $A m$ Rev Respir Dis 1961;83:566-7.

6 Dalhamn T. Studies on tracheal ciliary activity: special reference to the effects of cigarette smoke in living animals. Am Rev Respir Dis 1964;89:870-7.

7 Chand N, Pillar J, Diamantis W, Sofia RD. Inhibition of IgE-mediated allergic histamine release by azelastine and selected antiallergic drugs. Agent Actions 1985;16:318-22.

8 Katayama S, Tsunoda H, Sakuma Y, Kai H, Tanaka I Katayama $K$. Effect of azelastine on the release and action of leukotriene $\mathrm{C}_{4}$ and $\mathrm{D}_{4}$. Int Arch Allergy Appl Immunol 1987;83:284-9.

9 Taniguchi K, Takanaka K. Inhibitory effects of various drugs on phorbol myristate acetate and n-formyl methionyl leucyl phenylalanine induced $\mathrm{O}_{2}$-production methionyl leucyl phenylalanine induced $\mathrm{O}_{2}$-production in polymorphonuclear leukocytes. Biochem Pharmacol

10 Nakamura T, Nishizawa Y, Sato T, Yamato C. Effect of azelastine on the intracellular $\mathrm{Ca}^{2+}$ mobilization in guinea pig peritoneal macrophages. Eur $\mathcal{f}$ Pharmacol 1988;148:35-41.

11 Tanigawa $\mathrm{T}$, Honda $\mathrm{M}$, Miura $\mathrm{K}$. Effect of azelastine hydrochloride on vascular permeability reaction skin site in guinea pig. Arzneim-Forsch Drug Res 1981;31:1212-5.

12 Rose GG. A separable and multipurpose tissue culture chamber. Texas Rep Biol Med 1954;12:1074-83. 
13 Tamaoki J, Kondo $M$, Takizawa $\mathrm{T}$. Effect of cAMP on ciliary function in rabbit tracheal epithelial cells. $\mathcal{F} A p p$ Physiol 1989;66:1035-9.

14 Tamaoki J, Kondo M, Takizawa T. Adenosine-mediated cyclic AMP-dependent inhibition of ciliary activity in rabbit tracheal epithelium. Am Rev Respir Dis 1989;139:441-5.

15 Wanner A, Sielczak M, Mella JF, Abraham WM. Ciliary responsiveness in allergic and nonallergic airways. 7 Appl Physiol 1986;60:1967-71.

16 Brooker G, Harper JF, Terasaki WL, Moylan RD. Radioimmunoassay of cyclic AMP and cyclic GMP. Adv Cyclic Nucleotide Res 1979;10:1-33.

17 Lowry OH, Rosenbrough NJ, Farr AL, Randall RJ Protein measurement with the Folin phenol reagent. f Biol Chem 1951;193:265-75.

18 Villalón $M$, Hinds TR, Verdugo $P$. Stimulus-response coupling in mammalian ciliated cells: demonstration of two mechanisms of control for cytosolic $\left[\mathrm{Ca}^{2+}\right]$. Biophys f 1989;56:1255-8.

19 Kobayashi K, Tamaoki J, Sakai N, Chiyotani A, Takizawa $T$. Inhibition of ciliary activity by phorbol esters in rabbit epithelial cells. Lung 1989;167:277-84.

20 Chand N, Pillar J, Diamantis W, Perhach J, Sofia RD.
Inhibition of calcium ionophore (A23187)-stimulated histamine release from rat peritoneal mast cells by azelastine: implication for its mode of action. Eur $f$ Pharmacol 1983;96:227-33.

21 Nakamura T, Nishizawa Y, Sato T, Yamato C. Effect of azelastine on the intracellular $\mathrm{Ca}^{2+}$ mobilization in guinea pig peritoneal macrophages. Eur $\mathcal{f}$ Pharmacol 1988;148:35-41.

22 Akagi M, Mio M, Tanaka K, Kiniwa S. Mechanism of histamine release inhibition induced by azelastine. Pharmacometrics 1983;26:191-8.

23 Satir P. The role of axonemal components in ciliary motility. Comp Biochem Physiol 1989;94A:351-7.

24 Ross SM, Corrison S. Results of an analytical model of mucociliary pumping. F Appl Physiol 1974;37:333-40.

25 Jackowski JT, Szapfalusi ZS, Wanner DA, Seybold ZS, Sielczak MW, Lauredo IT, et al. Effects of $P$ aeruginosa-derived bacterial products on tracheal ciliary function: role of $\mathrm{O}_{2}$ radicals. $A m f$ Physiol 1991;260:L61-7

26 Berisha H, Foda H, Sakakibara H, Trotz M, Pakbaz H, Said SI. Vasoactive intestinal peptide prevents lung injury due to xanthine/xanthine oxidase. $\mathrm{Am} f \mathrm{Physiol}$ 1990;259:L151-5.

\section{Adventitia}

\section{The lung has no parenchyma}

In case this proposition seems too iconoclastic, I will restate it as follows: within the lung there is no tissue that can usefully be identified and named "parenchyma." I have urged for many years that the use of this word in relation to the lung is inappropriate and should be avoided. Avoidance is not difficult. George Simom managed to write an excellent textbook on lung radiology (Principles of chest $x$-ray diagnosis, 3rd edn. Butterworth, London, 1971) without once using the phrase "lung parenchyma". Fifteen years ago I confirmed in a formal study $(\mathrm{Br} \mathcal{F}$ Dis Chest 1978;72:1-12) that the meanings attached to this phrase by physicians and radiologists on both sides of the Atlantic are so varied that it is virtually meaningless. In many instances the word "parenchyma" can be omitted without altering the apparently intended meaning; in others it is necessary to ascertain the sense in which this word is being used. This sense is usually discordant with accepted usages of "parenchyma" in relation to other organs.

How did this verbal confusion arise? In relation to secreting glands or the liver, "parenchyma" refers to tissues composed of cells having functions specific to the organ concerned, as opposed to supporting and vascular tissues; this usage is unequivocal and, I believe, generally accepted. In the lung I suppose the cells that might be considered to be most organ specific are the type II pneumo- cytes, but it would be absurd to call them the lung parenchyma! The function of the lung depends not upon any one type of cell, but upon the structural and functional interaction of elements of its vasculature and airways to bring about orderly gas exchange in the alveoli. In general it seems that what most people mean when they speak of the lung parenchyma is its peripheral gas exchanging part. There is a fairly well defined demarcation at the ends of the terminal bronchioles between the part in which movement of gas is by diffusion both within airspaces and across alveolar membranes, and airways in which it is by ventilatory to and fro movement. It is now agreed that the part of the lung associated with a terminal bronchiole should be called an acinus; thus I think that what is usually meant by the "lung parenchyma" is the pulmonary acini. If you hear or read these words, consider first whether the word "parenchyma" contributes anything; it can often be eliminated without loss of meaning It may be evident from context that the intended reference is to the pulmonary acini, as opposed to the airways. If you do not think that anyone using these words has this meaning in mind, ask what he or she really means.

I recommend that this weasel word be eliminated from discourse concerning the lung.

G SCADDING 